\title{
Chromium Oxide Nanoparticle Impaired Osteogenesis and Cellular Response to Mechanical Stimulus
}

\author{
Jian Chen ${ }^{1, *}$ \\ Jing Ding ${ }^{1} *$ \\ Yuanhao $\mathrm{Wu}^{2}$ \\ Shuqiong Zhang ${ }^{3}$ \\ Naisheng Zheng ${ }^{3}$ \\ Junyao Yang $^{3}$ \\ Jing $X u^{\prime}$
}

'Department of Paediatric Orthopaedics, Xinhua Hospital, School of Medicine, Shanghai Jiao Tong University, Shanghai, 200092, People's Republic of China; ${ }^{2}$ Biodiscovery Institute, School of Pharmacy, University of Nottingham, Nottingham, UK; ${ }^{3}$ Department of Clinical Laboratory, Xinhua Hospital, School of Medicine, Shanghai Jiao Tong University, Shanghai, 200092, People's Republic of China

*These authors contributed equally to this work
Background: Release of metallic wear particles from hip replacement implants is closely associated with aseptic loosening that affects the functionality and survivorship of the prostheses. Chromium oxide nanoparticles (CrNPs) are the dominant form of the wear particles found in the periprosthetic tissues. Whether CrNPs play a role in the clinically observed particle-induced osteolysis, tissue inflammatory reactions and functional activities of human mesenchymal stem cells (MSCs) remain unknown.

Methods: A tibia-defect rat model, cytotoxicity assays and flow cytometry were applied to study the effect of CrNPs on MSCs survival and macrophage inflammatory response. Also, oscillatory fluid flow stimulation was used to analyse the osteogenic differentiation of MSCs while treated by CrNPs. In addition, the influence of CrNPs on MSC biomechanical properties was determined via atomic force microscope (AFM) and fluorescence microscopy.

Results: It was found that implantation of CrNPs significantly decreased bone formation in vivo. CrNPs had no obvious effects on inflammatory cytokines release of U937 macrophages. Additionally, CrNPs did not interfere with MSCs osteogenic differentiation under static culture. However, the upregulated osteogenic differentiation of MSCs due to fluid flow stimulation was reduced by CrNPs in a dose-dependent manner. Moreover, osteogenic gene expression of OPN, Cox 2 and Rnux 2 after mechanical stimulation was also decreased by CrNPs treatments. Furthermore, cell elasticity and adhesion force of MSCs were affected by CrNPs over 3 days of exposure. We further verified that these effects of CrNPs could be associated with its interruption on cell mechanical properties.

Conclusion: The results demonstrated that CrNPs impaired cellular response to mechanical stimulus and osteogenesis without noticeable effects on the survival of the human MSCs.

Keywords: chromium nanoparticle, human mesenchymal stem cells, osteogenesis, mechanical stimulation, cytoskeleton

\section{Introduction}

Total hip replacement is the most effective treatment for patients with end-stage hip diseases, which has acquired incremental popularity and the cases of procedures have continued to grow. ${ }^{1}$ However, with the increasing number of primary THA (total hip arthroplasty) operations performed, there is, on the other hand, a concomitant rise in the cases of hip revision required. ${ }^{2}$ Revision surgery is mainly scheduled for the patients with progressive pain or implant loosening. Release of the $\mathrm{CoCr}$ (cobalt and chromium) metallic wear particles and ions both from the bearing surfaces and the modular taper junction of the hip replacement implant are recognized to play a critical role in initiating
Correspondence: Junyao Yang; Jing Xu Email yangjunyao@xinhuamed.com.cn; xujing0I@xinhuamed.com.cn 
periprosthetic tissue reactions that affect the functionality and survivorship of the prostheses. ${ }^{3-6}$

MoM (Metal-on-Metal) hips have been reported to produce a huge quantity of nanoparticles. Metallic wear particulates from $\mathrm{CoCr}$ implants have been analysed directly in the surrounding periprosthetic tissues with the chemical analysis have shown that chromium oxide particles were the predominant constituent. ${ }^{7}$ For example, Campbell has reported that nearly $50 \%$ of the particles in the periprosthetic tissues were $\mathrm{CoCr}$ alloy particulates and the other half were mainly $\mathrm{Cr}$ oxide particles. ${ }^{8} \mathrm{Xia}$ et al analysed tissue biopsies harvested from patients undergoing MoM revision and reported that $\mathrm{Cr}$ particles were the main elements found in the tissues. ${ }^{9}$ In addition, wear and corrosion debris generated from the non-articulating surface, like taper junctions of MoM and MoP (Metal-on-Polyethylene) implants are also primarily $\mathrm{Cr}$ phosphate or oxide products, ${ }^{5,10}$ which is suggested to be caused by the rapid dissolution of Co content, thereby resulting in oxidized $\mathrm{Cr}$ particles of low solubility present in the tissue environment.

The effects of $\mathrm{Co}^{2+}$ or $\mathrm{Cr}^{3+}$ ions on immune cell apoptosis and cytokine/chemokine release have been profoundly documented. ${ }^{11-15}$ On the other hand, numerous in vitro and in vivo studies have investigated the biological effects of $\mathrm{CoCr}$ particles on macrophage and osteoblast, whereas there is limited knowledge about the effects of nanometresize chromium oxide particles. In a study by VanOs et al, the results show that $\mathrm{Cr}_{2} \mathrm{O}_{3}$ nanoparticles overall have rather minimal toxic effects on human macrophages in terms of cytokine release and they are cytotoxic only at rather high concentrations. ${ }^{16}$ Therefore, it seems that CrNPs were not the key player for the adverse tissue reaction and bone lesions around MoM prostheses. However, it remains unknown whether CrNPs had any detrimental effect on bone formation in vivo and bone-forming cells.

Osteogenesis by mesenchymal stromal cells (MSCs) is vital for normal bone healing and successful osseointegration of implants. It is now clear that MSCs play an indispensable role in wear particles-related aseptic implant loosening. ${ }^{17}$ MSCs play a crucial role in bone regeneration by regulating osteoblast formation via many microenvironmental signals. ${ }^{18}$ The MSC-to-osteoblast differentiation dependents on bio-chemical cues ${ }^{19}$ such as soluble mediators including Wnt, bone morphogenetic protein (BMP) and fibroblast growth factors (FGFs) that actively support osteogenesis. ${ }^{20}$ On the other hand, MSCs are also found to experience mechanical stimuli that includes shear stress or oscillatory fluid flow to encourage cell proliferation and differentiation. ${ }^{21}$ Therefore, it is of great interest and implication to understand whether wear particles, especially CrNPs, had a direct bio-mechanical effect on the MSCs. Hence, the effect of CrNPs on bone formation in vivo was investigated in this study. Also, its influence on cell survival, bio-mechanical cues-induced osteogenesis of MSCs and underlying mechanism was examined.

\section{Materials and Methods Human U937 Macrophage and MSCs Culture and Preparation}

U937 cell line (donation from Dr. Akihisa Mitani, Imperial College London, UK; cells were authenticated by STR profile and approved by institutional research ethics committee of Xinhua Hospital, School of Medicine, Shanghai Jiao Tong University) were cultured in RPMI 1640 medium (Sigma Aldrich) with 10\% FBS, $1.9 \mathrm{mM}$ L-glutamine, 96 $\mathrm{U} / \mathrm{mL}$ penicillin and $96 \mu \mathrm{g} / \mathrm{mL}$ streptomycin in a humid incubator with $5 \% \mathrm{CO}_{2}$ at $37^{\circ} \mathrm{C}$. To induce its differentiation into macrophage-like cells, U937 cells were treated with PMA (50 ng/mL, Sigma Aldrich) for 2 days and rest for another 24h in RPMI 1640 medium prior to experiments.

Human MSCs were isolated from bone marrow aspirate (ALLCELLS, Lot No: BM2893). The cells were grown in $\alpha$-MEM (Gibco, Thermo Fisher Scientific, UK) supplemented with $10 \%$ foetal calf serum (FCS, Sigma), amphotericin B $(0.625 \mu \mathrm{g} / \mathrm{mL}$, Sigma $), 100 \mathrm{IU} / \mathrm{mL}$ penicillin, $100 \mu \mathrm{g} / \mathrm{mL}$ streptomycin (Sigma) and L-ascorbic acid 2-phosphate solution (50 mg/mL, Sigma, UK). The cells were maintained at $37^{\circ} \mathrm{C}$ in a humidified atmosphere containing 5\% $\mathrm{CO}_{2}$. Cells between passages 2 and 4 were used for experiments. For osteogenic differentiation, cell culture medium was also supplemented with dexamethasone $(0.3 \mathrm{nM})$ and $\beta$-Glycerolphosphate $(10 \mathrm{mM})$, which support osteogenesis while allowing greater scope to study the effect of fluid flow stimulation on MSC osteogenic differentiation when treated by CrNPs.

\section{Preparation of Chromium Nanoparticles}

CrNPs from American Elements (Los Angeles, CA, USA) were in the form of chromium oxide $\left(\mathrm{Cr}_{2} \mathrm{O}_{3}\right)$ nanoparticles. The diameter of the particles ranges from 10 to $30 \mathrm{~nm}$ according to the manufacturer data. The CrNPs were cleaned in $100 \%$ ethanol for sterilisation followed by resuspension in sterile DI water at a concentration of $1 \mathrm{mg} / \mathrm{mL}$ prior to experiment. 


\section{Transmission Electron Microscopy (TEM)}

CrNPs were prepared by dispersing in sodium acetate buffer at a concentration of $1 \mathrm{~g} / \mathrm{L}$ followed by sonication. The suspensions were then pipetted onto 400 -square mesh carbon-coated TEM grids. The solvent was allowed to evaporate at ambient laboratory conditions for $20 \mathrm{~min}$ before imaging. TEM images were recorded in bright field mode. The specimens were imaged with an electron microscope (H7600; Hitachi, Tokyo, Japan) operating at $100 \mathrm{kV}$.

\section{Particle Characterization}

Hydrodynamic diameter of the CrNPs $(10 \mu \mathrm{g} / \mathrm{mL})$ was measured via dynamic light scattering (DLS) in a-MEM cell culture medium using a Malvern Zetasizer (Malvern, UK) at a temperature of $25^{\circ} \mathrm{C}$.

\section{In vivo Osteogenesis Animal Model}

All animal procedures were performed in accordance with the guidelines for the care and use of laboratory animals approved by Xinhua Hospital, School of Medicine, Shanghai Jiao Tong University (XHEC-NSFC-2019-194) and the ethical review board of Xinhua Hospital, School of Medicine. The chromium nanoparticles were sterilized before grafting into 6-weeks-old female SD rats at the tibia defects site. Six animals were used for each group. A liner skin incision in the proximal tibia was made to expose the proximal tibia shaft. A $2.5 \mathrm{~mm}$-diameter anterior cortical defect perpendicular to the shaft axis was then made using surgical drill. $50 \mu \mathrm{L}$ of PBS with or without CrNPs $(0.1 \mathrm{mg})$ was placed into the defect sites according to group allocation. The rats were sacrificed at 4 weeks after operation.

\section{$\mu \mathrm{CT}$ Analysis}

Embedded specimens were scanned by $\mu \mathrm{CT}$ (SkyScan 1172; Bruker-micro-CT, Kontich, Belgium). The scanned images were reconstructed by image analysis software (CTanalyzer; Skyscan). The Bone mineral density (BMD) and total bone volume (BV)/total tissue volume (TV) were measured to analyse the bone regeneration in the defect sites.

\section{Histological Staining}

The harvested samples were first decalcified in 10\% ethylene diaminetetraacetic acid (EDTA) and dehydrated in a graded ethanol prior to embedding in paraffin. Then, longitudinal serial sections of $5 \mathrm{~mm}$ were cut and mounted on microscope slides. Each sample was stained with haematoxylin and eosin staining (H\&E).

\section{Cytokine Profiling}

Cytokines released in the cell culture media were measured with Proteome Profiler Human Cytokine Array Kit (R\&D system, USA). This assay is capable of detecting a panel of 36 chemokines as shown in Table 1 . The array membranes were first immersed with conditioned media followed by 3 washes. After incubation with first and secondary conjugated antibodies, the sample was then exposed to HRP substrate for half an hour. The intensity of the reaction was quantified and analysed as previously reported. ${ }^{22}$

\section{Cell Viability Test}

Proliferation of MSCs was examined by CellTiter $96^{\circledR}$ Cell Proliferation Assay (MTS assay, Promega). MSCs at $5 \times 10^{4}$ cells/well were seeded and treated for 3 days with $5-50 \mu \mathrm{g} / \mathrm{mL}$ of CrNPs. Following exposures, cell culture supernatants were harvested and analysed as previously depicted. ${ }^{22}$ Five replicates of each exposure were tested with the assay repeated in three separate experiments.

\section{Fluorescence-Activated Cell Sorting (FACS) Analysis}

Apoptosis of MSCs was determined via FACS utilising a FITC Annexin V Apoptosis Detection Kit with PI (Biolegend, UK) as mentioned in our previous study. ${ }^{22}$

Table I Cytokines Detected with the Proteome Profiler Human Cytokine Array

\begin{tabular}{|l|l|l|l|l|l|}
\hline C5a & IL-4 & CCL4 & ICAM-I & IL-32 alpha & IL-I3 \\
\hline G-CSF & IL-6 & CXCLI2 & IL-I alpha & CXCLII & IL-I7 \\
\hline CXCLI & IL-10 & TNF-alpha & IL-I ra & MIF & IL-23 \\
\hline CCLI & IL-12 P70 & TREM-I & IL-2 & CCL3 & IL-27 \\
\hline CD40 ligand & IL-5 & CCL5 & IFN-gamma & CXCLI0 & IL-I6 \\
\hline GM-CSF & IL-8 & Serpin EI & IL-I beta & MCP-I & IL-I7E \\
\hline
\end{tabular}




\section{Atomic Force Microscopy (AFM)}

The mechanical properties of MSCs treated by CrNPs was measured with Atomic force microscopy (AFM). JPK NanoWizard 4 system with an inverted microscope (Axio Observer Z1, Zeiss) was utilized for the experiment. ${ }^{23}$

\section{Oscillatory Fluid Flow Mechanical}

\section{Stimulation}

Oscillatory fluid flow was achieved by using the Flexcell Streamer parallel plate flow chamber system Flexcell Streamer System (FX4000, Flexcell International, USA) as illustrated. ${ }^{24}$ The apparatus was placed in an incubator for the duration of the experimental maintained at $37^{\circ} \mathrm{C}$ under $5 \%$ $\mathrm{CO}_{2}$. The cells were seeded on the type I collagen-coated glass slides (Flexcell, USA) for $24 \mathrm{~h}$ prior to experiment. To determine the influence of CrNPs on osteogenic responses and osteogenic lineage commitment of MSCs, the flow regimes applied in this research comprise no flow control samples, short- and long-term stimulation with or without CrNPs treatment. The MSCs were exposed to a $4 \mathrm{~h}$ of short-term stimulation or a long-term fluid shear on Days 3, 4, 6, 7 for $4 \mathrm{~h}$ a day followed by an additional 7 days of static culture. The flow regime consisted of a fluid shear of magnitude at $1 \mathrm{~Pa}$ and with a frequency of oscillation of $1 \mathrm{~Hz}$ as depicted in the results section. No flow control slides were also loaded into flow chambers, while fluid flow was not applied.

\section{Quantitative Real-Time PCR}

After oscillatory fluid flow mechanical stimulation, RNA sample from lysed MSCs was transcribed into cDNA using High Capacity cDNA kit (Life Technologies) according to manufacture protocol. Quantitative polymerase chain reaction was conducted using SYBR Select Master mix (Thermo Fisher, UK). The expression of 18S (18S ribosomal RNA), Cox2 (Cyclooxygenase-2), OPN (osteopontin) and Runx2 (Runt-related transcription factor 2) was quantified using primers detailed in Table 2 (Sigma, UK). ABI7500 Fast Real Time PCR machine was used for the amplification. Sample was normalized to reference gene $18 \mathrm{~S}$.

\section{Osteogenic Assays}

For ALP staining, the samples were first fixed for $30 \mathrm{~min}$ with 95\% cold ethanol followed by three washes in PBS. A leukocyte alkaline phosphatase kit (Sigma-Aldrich, UK) was used and ALP-positive cells were stained blue. ALP activity measurement. ALP activity was also determined using an ALP assay kit (Abcam, US) according to the manufacturer's instructions. Briefly, cells were washed with ice-cold PBS, lysed with $0.5 \%$ Triton X-100, and centrifuged. ALP assay was performed in alkaline buffer solution (1.5 M, $\mathrm{pH}$ 10.3) containing $10 \mathrm{mM}$ p-nitrophenyl phosphate as a substrate. Following the addition of the stop solution, the optical density was measured in a microplate reader at $405 \mathrm{~nm}$. ALP activity was normalized with the value of DNA content.

\section{Immunofluorescence Staining and Microscopy}

The cells were fixed and permeabilized as previously depicted. ${ }^{25}$ Vinculin, the adhesion protein, was labelled with mouse monoclonal anti-vinculin primary antibody (1:200, Sigma, USA) and conjugated anti-Mouse IgG Secondary Antibody (1:1000, Invitrogen, USA). To label the F-actin structure, the samples were stained with phalloidin (Molecular Probes, USA) with cell nuclei visualized by incubation with 1 DAPI ( $5 \mu \mathrm{g} / \mathrm{m}$, Dojindo, USA). Slides were then imaged with a fluorescence microscope (SP5, Leica, Germany) with x63 objective.

\section{Data Analysis and Statistics}

All data were expressed as the mean + SEM of at least three independent experiments. The statistical differences between groups were analysed with Student's $t$-test (SPSS Inc., Chicago, IL, USA). A value of $p<0.05$ was considered statistically significant.

\section{Results}

\section{Particle Characterizations}

The morphology of the CrNPs used in this study was observed using TEM. Most of the nanoparticles were polygonal with some of them were spherical and fused into large agglomerates (Figure 1A). The CrNPs have a pristine size of $10-30 \mathrm{~nm}$ in diameter according to manufacturer data. Representative data of CrNPs size distribution measured by Zetasizer in intensity is shown in Figure 1B. The hydrodynamic size of CrNPs was affected by exposure to serum-contained culture medium, which agglomerated in the medium with main peak around 320 $\mathrm{nm}$ (average: $267.7 \mathrm{~nm}$ ). The commercially available CrNPs used in this study are clinically relevant. The characteristics of CrNPs are consistent with the size, morphology, and chemical composition of metal particles collected from the tissues around MoM hips in vivo ${ }^{26,27}$ and the particles generated by hip simulators in vitro by previous studies. $^{28,29}$ 


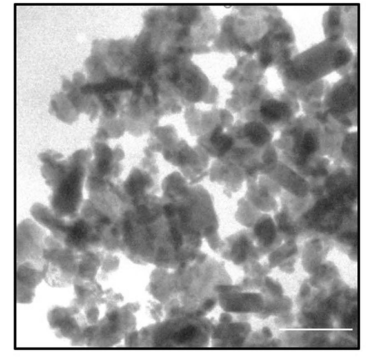

A

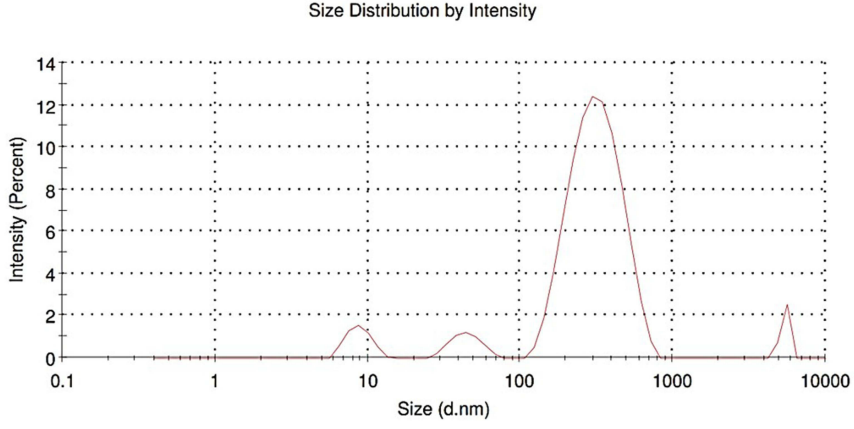

B

Figure I Characterization of the NPs and intracellular distribution. (A) TEM images of CrNPs. Scale bar indicate $100 \mathrm{~nm}$. (B) Intensity-density size distribution of CrNPs in complete cell culture medium measured by Zetasizer.

\section{Effect of CrNPs on Osteogenesis in vivo}

To investigate the role of CrNPs in aseptic osteolysis, the effect of CrNPs on osteogenesis in vivo was analysed via a tibia-defect animal model (Figure 2A). The concentration of the CrNPs used in this study was based on previous in vivo researches, ${ }^{30}$ which ranges from less than $1 \mathrm{mg} /$ animal to as high as $30 \mathrm{mg}$ /animal and we applied a relatively moderate dose of CrNPs. Histological evaluation was performed by H\&E staining on the bone defect sites of proximal tibia 4 weeks after implantation of CrNPs. The morphology and bone trabecular tended to be distinct between control group and CrNPs-treated group. Cross-section staining of the defect site (Arrows, Figure 2B and C) revealed higher amounts of newly formed bone and more mature trabecular bone in the control rat (Figure 2B) compared with the CrNPs-treated counterpart (Figure 2C). The defect of the tibia from the control mice was almost filled with newly formed cortical bone (Figure 2B), which represented a normal bone healing process. $\mu \mathrm{CT}$ images of different views and 3D reconstruction (Figure 2D and E) were used to further image and quantify new bone formation at week 4 after tibia defect surgery with (Figure 2E) or without particle implantation (Figure 2D). Trabecular numbers, bone mineral density (BMD) and total bone volume (BV)/total tissue volume (TV) were used as parameters for quantitative analysis. Four weeks after surgery, both indicators of bone formation in the CrNPs group were significantly lower than in the control group as shown (Figure $2 \mathrm{~F}-\mathrm{H}$ ), which suggested the detrimental effects of CrNPs on bone regeneration in vivo.

\section{Effect of CrNPs on Inflammatory Response of Macrophages}

The cellular response to metal wear particles involves various cell types including immune cells and bone-forming cells. It has been well established that CoCrMo wear particles initiate an immune response inducing osteolysis and aseptic loosening of the implant. ${ }^{3,11}$ To investigate whether the adverse effects of CrNPs on bone regeneration in vivo was resulted from local tissue inflammation, its effect on the inflammatory response of macrophages was analysed via a cytokine array. The representative images of the cytokine profile of U937 macrophages cultured with or without CrNPs for $72 \mathrm{~h}$ are shown in Figure 3A and B. $5-50 \mu \mathrm{g} / \mathrm{mL}$ of CrNPs used in the tests had no cytotoxic effect on U937 macrophages according to our previous results. ${ }^{25}$ According to the results (Figure 3C), no statistically significant difference of the cytokines detectable in the cell culture medium between control and treated cells could be found. Additionally, CrNPs did not stimulate the release of IL1- $\beta$ (interleukin 1 beta), IFN- $\gamma$ (interferon-gamma) and IL-6 (interleukin 6), which were found to be important mediators of aseptic implant loosening and osteolysis. ${ }^{31}$ Therefore, it seems that the decreased bone regeneration in vivo after CrNPs implantation was not due to the local inflammatory response of macrophages exposed to the particles.

\section{Effect of CrNPs on Human MSCs Apoptosis and Proliferation}

MSCs are the critical for maintaining osseous tissue integrity. Accumulating evidence revealed that wear particles significantly impair MSC-to-osteoblast differentiation and 
Table 2 Sequence for the Primers

\begin{tabular}{|l|l|}
\hline Gene & Sequence \\
\hline I8S & 5'-GTAACCCGTTGAACCCCATT-3' \\
\hline & 3'-CCATCCAATCGGTAGTAGVG-5' \\
\hline Cox2 & 5'-ACTCATAGGAGAGACTATCAAG-3' \\
\hline & 3'-GAGTGTGTTGAATTCAGAGG-5' \\
\hline OPN & 5'-GGATGAATCTGACGAATCTC-3' \\
\hline & 3'-GCATCAGGATACTGTTATC-5' \\
\hline Rnux2 & 5'-AACCCACGAATGCACTATCCA-3 \\
\hline & 3'-CGGACATACCGAGGGACATG-5' \\
\hline
\end{tabular}

reduce new bone formation. To determine whether the decreased bone regeneration in vivo (Figure 2) was related to the reduction of MSCs viability or osteogenic events due to the cytotoxic effects of CrNPs, the apoptosis rate of MSCs was analysed via Annexin V-FITC/PI assay with FACS after $24 \mathrm{~h}$ of exposure. $50 \mu \mathrm{M}$ of CCCP (Carbonyl cyanide 3-chlorophenylhydrazone) was used an apoptosis inducer. As shown in Figure 4A-F, after $24 \mathrm{~h}$ of treatment, the amount of the percentages of apoptotic cells between untreated and treated cells with 5-50 $\mu \mathrm{g}$ of CrNPs was not statistically different, which indicated that CrNPs had minimal impact on MSCs viability. Further, the proliferation of MSCs in the presence of CrNPs was assessed during 3 days of culture, which was investigated using a CellTiter MTS cell proliferation assay. According to the results, CrNPs at concentrations ranging from 5 to 50 $\mu \mathrm{g}$ had no statistically significant effects on human MSC proliferation over $72 \mathrm{~h}$ of treatment (Figure 4G-I), which is consistent with previous reported results by other researchers. ${ }^{16}$ In the following experiments, we continued to investigate the effects of CrNPs on cell activities by using these concentrations of particles.

\section{Effect of CrNPs on Human MSCs Osteogenic Lineage Commitment}

Dysfunctions of MSC lineage commitment have been related to osteoporosis and many other metabolic bone diseases. ${ }^{18}$ To further unravel the mechanism of the detrimental effects of CrNPs on osteogenesis, human MSCs were exposed to CrNPs under 2 weeks of static culture or with oscillatory fluid flow stimulation as shown in Figure 5A, which mimic the physical stimuli loaded on
MSCs in the bone marrow cavity in vivo. MSCs were stained for alkaline phosphatase (ALP) and ALP activity was measured to assess osteogenic differentiation after 2 weeks of treatment. As indicated in Figure 5B and C, the results showed that CrNPs ranging from 5-50 $\mu \mathrm{g}$ has minimal effects on MSCs osteogenesis under static culture. It could also be noted that fluid flow stimulation markedly enhanced ALP expression and activity for untreated MSCs after 2 weeks of culture upon mechanical stimulation (Figure 5D and E). However, this stimulating effect was reduced by CrNPs in a dose-dependent manner (Figure 5E). ALP quantitation analysis showed that the osteogenic differentiation of MSCs exposed to fluid flow was decreased by approximately $40 \%$ with $5 \mu \mathrm{g}$ of CrNPs treatment and the enhanced osteogenic differentiation was not found when the cells were exposed to $20 \mu \mathrm{g}$ and $50 \mu \mathrm{g}$ of CrNPs (Figure 5E). We further investigated whether CrNPs affect early osteogenic gene expression under fluid shear by measuring OPN, Cox 2 and Runx 2 mRNA expression after treatment. It was evident that osteogenic gene expression in MSCs was significantly increased in response to fluid flow when compared to the control (static culture condition). After $2 \mathrm{~h}$ of shear stress at a magnitude of $2 \mathrm{~Pa}$, the mRNA expression of OPN, Cox 2 and Runx2 was notably upregulated (Figure $5 \mathrm{~F}-\mathrm{H}$ ). While the upregulation of osteogenic gene expression could be inhibited with the addition of CrNPs. The reduction was most notable for the cell exposed to the highest amount of CrNPs. Therefore, although CrNPs have no apparent effect on human MSCs osteogenic differentiation under static condition, osteogenesis was greatly affected by CrNPs when the cells were cultured under fluid flow stimulation, which indicated that CrNPs had a negative influence on MSCs response to mechanical stimulus thereby inhibiting its osteogenic differentiation under fluid flow.

\section{Effect of CrNPs on Human MSCs Mechanical Properties and Cytoskeleton}

It has been shown that osteogenic differentiation of human MSCs under mechanical stimulus is mediated by cytoskeletal arrangement. ${ }^{32,33}$ Therefore, we hypothesized that mechanical properties and cytoskeleton integrity of the MSCs could be influenced by CrNPs. Mechanical property alteration of MSCs subjected to CrNPs was monitored using AFM over 3 days of treatments. Cells were exposed to CrNPs over 3 days at different concentrations ranging from 5 to $50 \mu \mathrm{g} / \mathrm{mL}$. For cells exposed to 20 or $50 \mu \mathrm{g} / \mathrm{mL}$ of CrNPs (Figure 6A), 
A

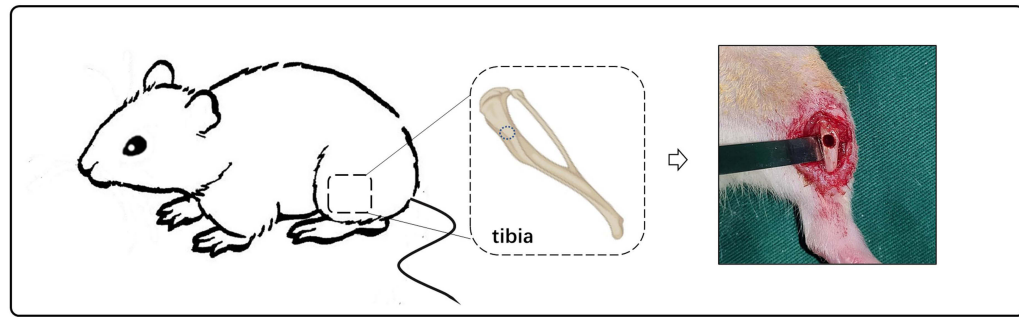

B

C

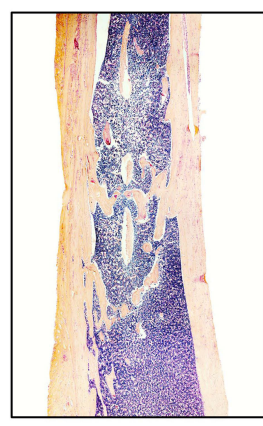

D

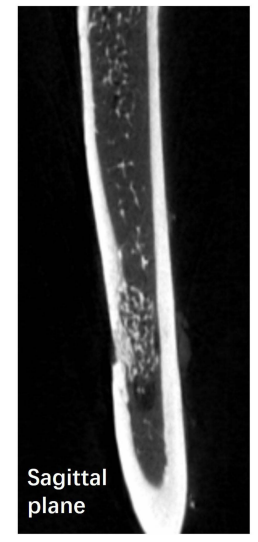

F

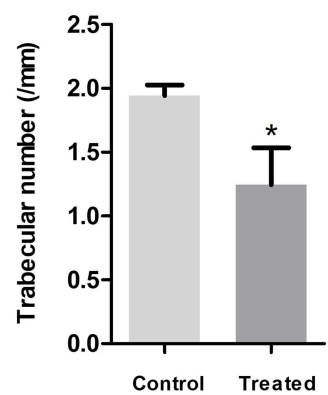

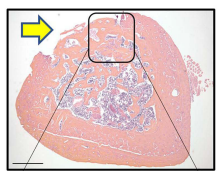

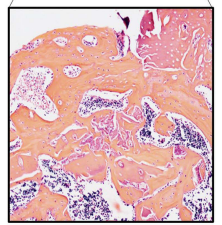

4.

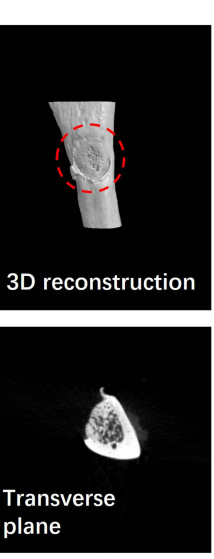

$\mathbf{G}$

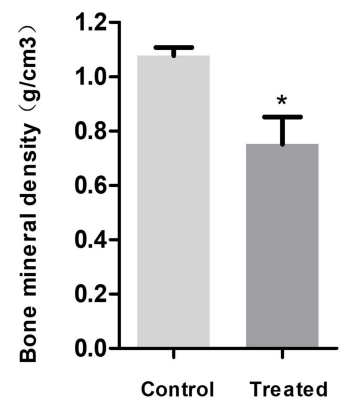

E
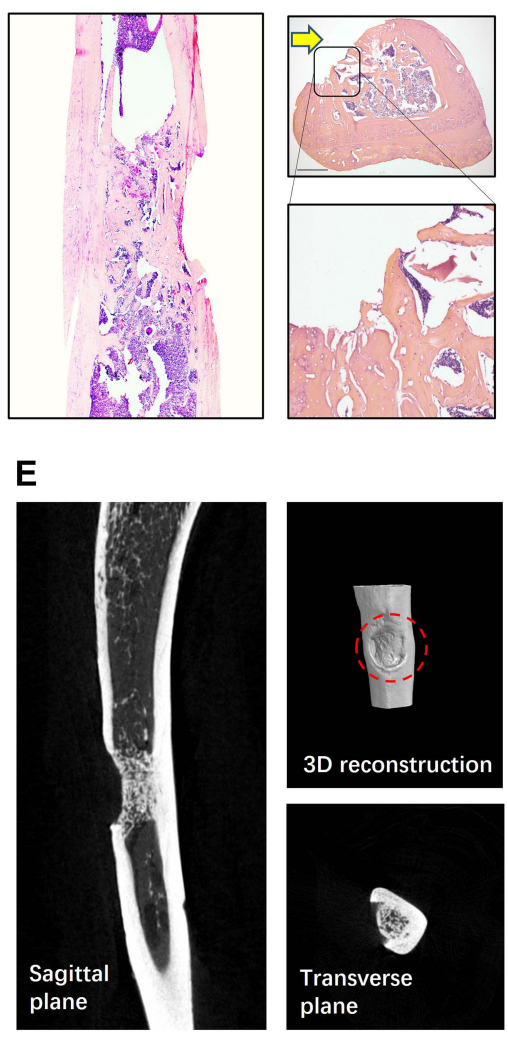

H

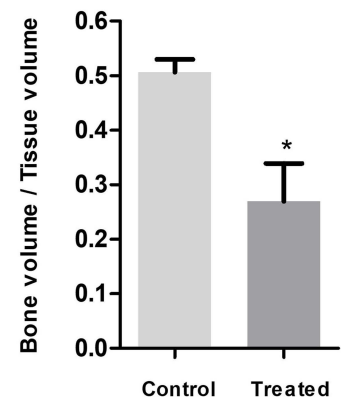

Figure 2 (A) Illustration of the in vivo osteogenesis animal model. Representative haematoxylin and eosin-stained histological morphology of the harvested tibia and cortical bone defects for control (B) and treated rat (C). Arrows indicate defected sites. Scale bar indicate I mm. Different planes of $\mu$ CT and 3D reconstruction images of the tibia for control (D) and treated rat (E). Dotted circles indicate defected sites. (F-H) Trabecular numbers, Bone mineral density (BMD) and bone volume (BV)/ tissue volume (TV) of new bone formation in the defect areas after 4 weeks (Bars represent mean + SEM; unpaired $t$-test, $\mathrm{n}=6$ per group; * $<$ < 0.05 ). 
A
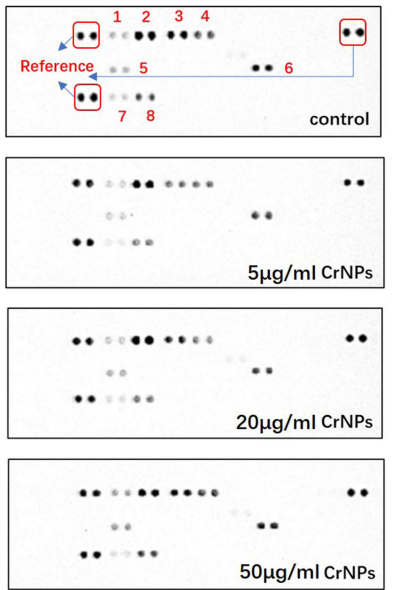

B

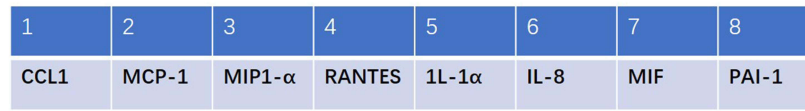

C

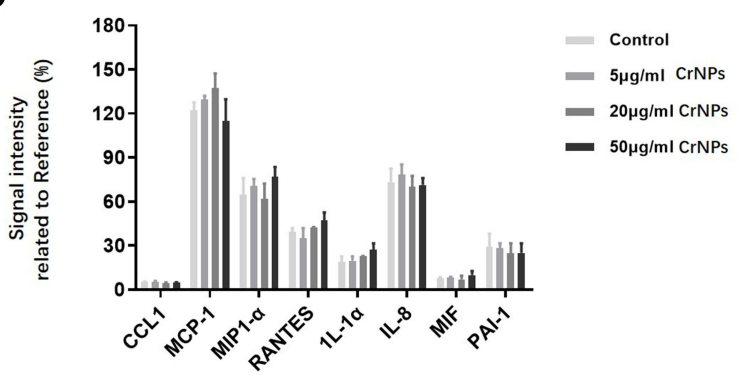

Figure 3 (A) Representative images of the stained membrane detecting cytokines released into the cell culture medium from different treatment groups. (B) list of detectable cytokines shown in (A). (C) Signal intensity of measurable cytokines from (A) showing mean \pm SEM. Data was from 3 independent experiments; unpaired Student's t-test.

a reduction in cell elasticity was identified after one day of exposure compared to MSCs that were not treated by any particles. The elasticity of MSCs not exposed to nanoparticles was about $3.4 \mathrm{kPa}$ and decreased to about $2.0 \mathrm{kPa}$ after 2 days and approximately $1.5 \mathrm{kPa}$ after 3 days of treatment with $50 \mu \mathrm{g} / \mathrm{mL}$ of CrNPs (Figure $6 \mathrm{~B}$ and $\mathrm{C}$ ). In addition, another important mechanical property of the cells investigated was the adhesion force of the MSCs pre- and postexposure to CrNPs. After 2 days of exposure to $50 \mu \mathrm{g} / \mathrm{mL}$ of CrNPs, MSCs exhibited much smaller adhesion forces compared with untreated cells (Figure 6E and F). CrNPs generally did not cause significant variation in the adhesion forces of MSCs for exposure up to 3 days at concentration lower than $50 \mu \mathrm{g} / \mathrm{mL}$. However, there was a general decrease in the mean adhesion force when the cells were exposed to incremental amount of CrNPs. Moreover, immunofluorescent staining demonstrated the differences in F-actin expression of the cells treated by CrNPs $(50 \mu \mathrm{g} / \mathrm{mL})$, in which the F-actin structures were more scattered and less aligned compared with untreated cells (Figure 6G). To further verify the associations between $\mathrm{Cr}$-mediated cell mechanics alteration and osteogenic response, we applied Cytochalasin B $(2 \mu \mathrm{M})$ to disrupt F-actin structure and PF562271 (5 $\mu \mathrm{M})$, a reversible inhibitor of focal adhesion kinase (FAK) that regulate the generation of cell adhesive forces to investigate their effects on cellular change under fluid flow. It is obvious that both Cytochalasin B and PF562271 induced marked cytoskeleton alteration, in which MSCs become less spread and spherical when treated by PF562271 for 24h (Figure 6G) or lost filamentous cytoplasmic and membrane-actin structures when exposed to Cytochalasin B for $3 \mathrm{~h}$ (Figure 6G). We then investigated the influence of these treatments on MSCs osteogenic gene expression under fluid shear. It was apparent that OPN, Cox2 and Runx 2 mRNA expression in MSCs was significantly downregulated when treated by Cytochalasin B and PF562271 before exposure to mechanical stimulus (Figure $6 \mathrm{H}$ and I-J). Cytochalasin B caused a dramatic change F-actin structures of MSCs and led to a complete inhibition of fluid flow-induced osteogenic response. In a word, our results indicated that the effects of CrNPs on flow-induced osteogenic differentiation could be associated with its interruption on cell mechanics, especially on cytoskeleton properties and cell adhesion force generation.

\section{Discussion}

Chromium oxide particles in the nanometer-size range are the predominant form of particles produced by MoM implants. However, the biological reactions to the CrNPs have not been examined specifically. MSCs serve as the key cells that play a critical role in mechanosensing and bone remodelling. ${ }^{34}$ However, little is known about the impact of clinically relevant CrNPs exposure on this cell population. Here, we unravelled that exposure to CrNPs was detrimental to osteogenesis and MSCs physiology. The impaired capacity of new bone formation due to CrNPs exposure was first verified by a one-month in vivo tibia defect animal model (Figure 2). 
A

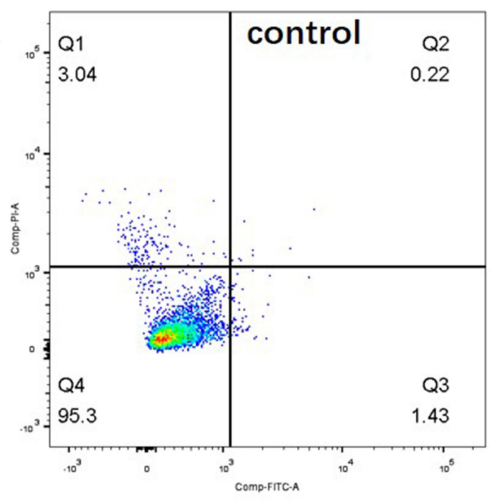

D

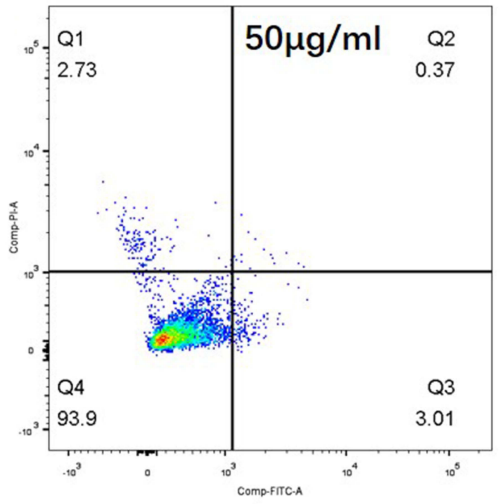

G

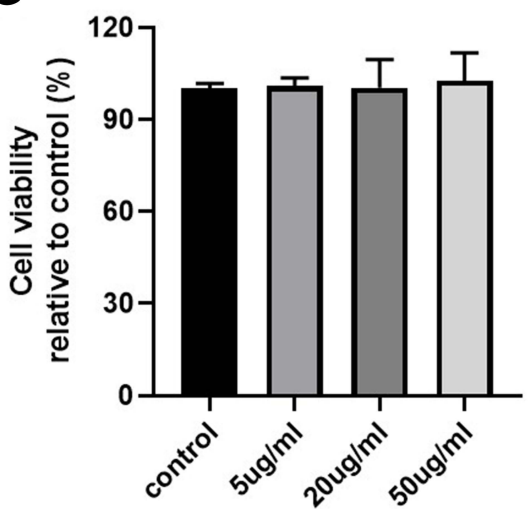

B
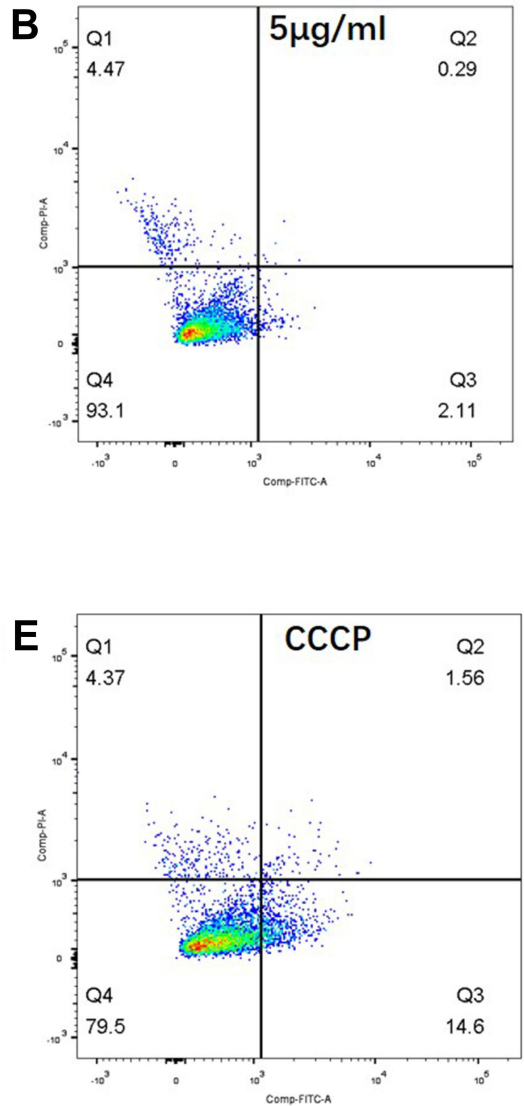

H

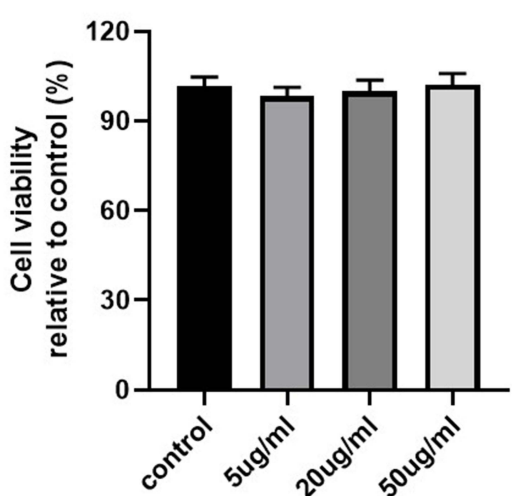

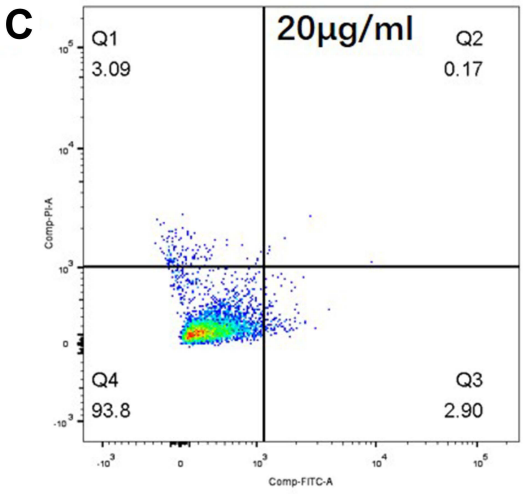

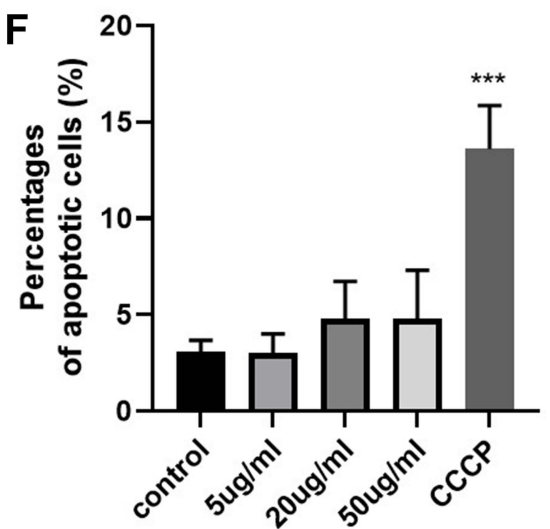

I

Day 3

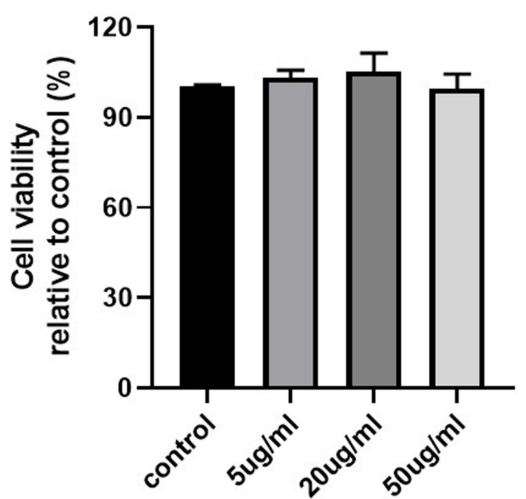

Figure 4 (A-E) Representative results of apoptosis of human MSCs exposed to CrNPs assessed by Annexin V-FITC/PI assay via FACS. (F) Quantification of apoptotic cells from different treatments. (G-I) The effect of CrNPs on human MSCs proliferation capacity grown in tissue culture plates. Data represents mean + SEM from 3 independent experiments with 5 replicates for each condition. unpaired Student's $t$-test. ${ }^{* * *} \mathrm{p}<0.00 \mathrm{I}$.

The response to CrNPs in vivo involves various different cell types. We first investigated the inflammatory reactions of macrophages exposed to CrNPs via a cytokine array, the results showed that CrNPs had no obvious effect on the release of inflammatory mediators from U937 macrophages. Further, cell apoptosis and metabolism experiments were applied to investigate whether the decreased osteogenesis was caused by the cytotoxicity of CrNPs on human MSCs, which plays a critical role in bone regeneration and osteolysis. According to the results, cell proliferation and viability was not significantly different between control and treated cells over $72 \mathrm{~h}$ of exposure to CrNPs, which is consistent with previous reports that 

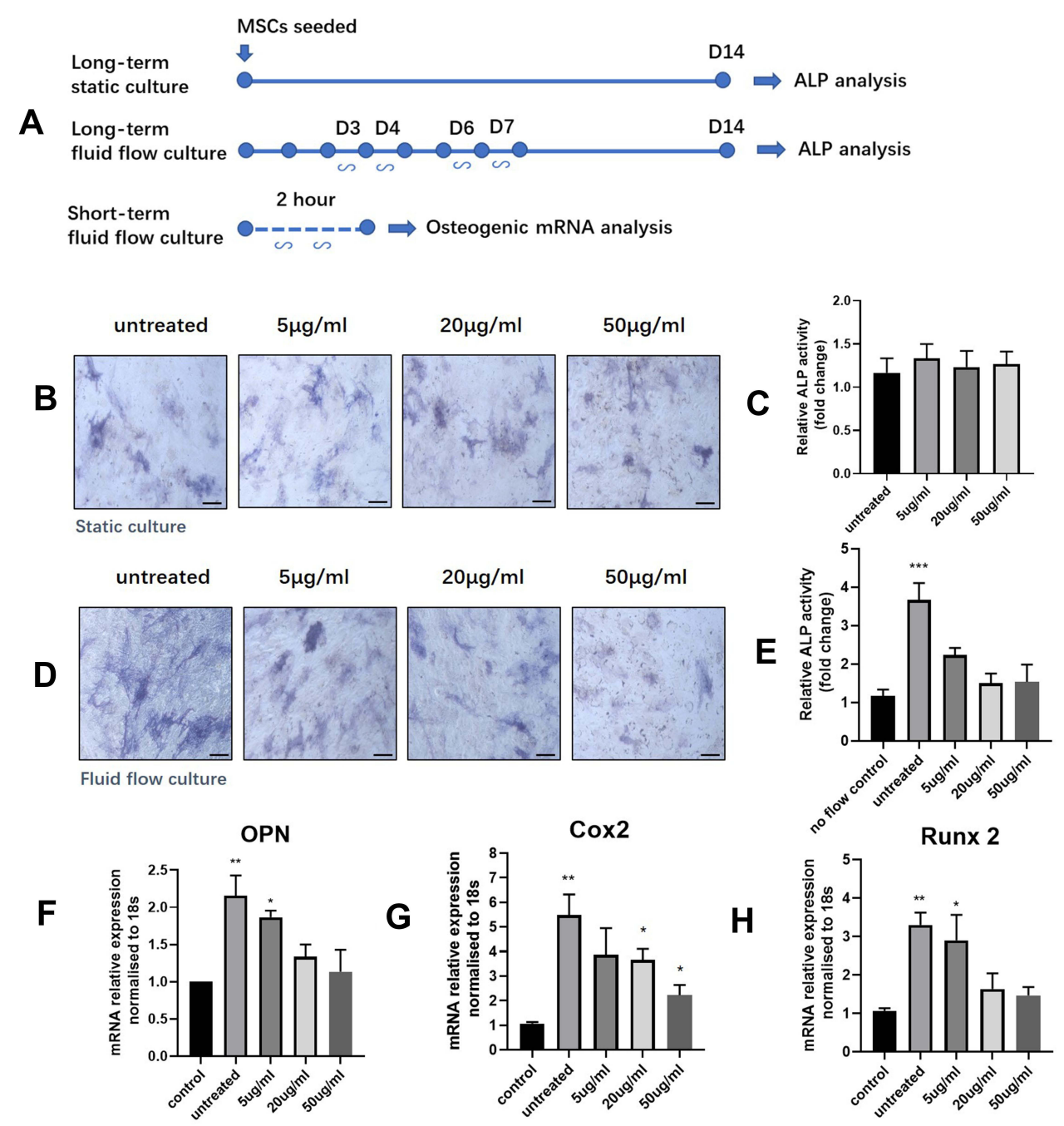

Figure 5 (A) Oscillatory fluid flow experimental timelines. MSCs were subjected to 3 separated regimens to study the effect of CrNPs on MSCs osteogenesis in vitro. Osteogenic differentiation of the MSCs under static and fluid-flow culture was visualized by ALP staining (B and $\mathbf{D})$ and the ALP activities were quantified in (C and $\mathbf{E}$ ). Undifferentiated MSCs (ALP negative) are colourless or faintly bluish, while MSC-derived osteoblasts (ALP positive) are dark blue-violet. Effect of CrNPs on early mRNA expression of osteogenic genes OPN (F), Cox2 $(\mathbf{G})$ and Runx2 $(\mathbf{H})$ under fluid flow. Bars represent mean + SEM; Statistically significant differences are indicated relative to untreated cells or control cells (untreated and under no-flow condition) based on unpaired $t$-test, ${ }^{*} \mathrm{p}<0.05$, ${ }^{* *} \mathrm{p}<0.0 \mathrm{I}$, ${ }^{* * *} \mathrm{p}<0.00 \mathrm{I}$.

demonstrated a minimal cytotoxic effect of $\mathrm{Cr}^{3+}$ and $\mathrm{Cr}_{2} \mathrm{O}_{3}$ particles even at high concentrations. ${ }^{16}$ Studies investigating the biological response to metal particles produced by MoM implants have focused predominantly on the effects of $\mathrm{CoCr}$ particles. Most of these studies have reported obvious decreases in cell viability and increased cell apoptosis due to cobalt element. ${ }^{11,35}$ This study demonstrates that chromium oxide particles have minimal toxic effects on human MSCs.

Although MSCs survival was not affected by the CrNPs, they are sensitive to these metal debris under mechanical stimulus. We showed for the first time that the mechanical properties of MSCs, including cell elasticity and adhesion forces, were affected in the presence of CrNPs, which could contribute to the impaired osteogenesis capacity of MSCs in vitro and in vivo. To investigate the effects of CrNPs on human MSCs osteogenesis, the cells were first exposed to CrNPs under static culture for 2 weeks. The results showed that CrNPs ranging from 5 to $50 \mu \mathrm{g}$ had minimal effects on MSCs osteogenesis (Figure 5B) measured by ALP activities and staining. Mechanical loading plays an indispensable role in maintaining the architecture and function in skeleton system. ${ }^{36}$ Skeletal loading from weight-bearing activities could produces interstitial fluid flow in the intramedullary cavity and within the cortices on cells resident in the tissue. ${ }^{37,38}$ Progenitor cells, such as MSCs that could differentiate into osteoblasts, serve as a natural source of functional osteoblasts during mechanical loading. ${ }^{19}$ It has been well established that mechanical stimulation promotes osteogenic lineage commitment of MSCs, which leads to enhanced bone formation. ${ }^{32,39}$ In vitro research have shown that mechanical 


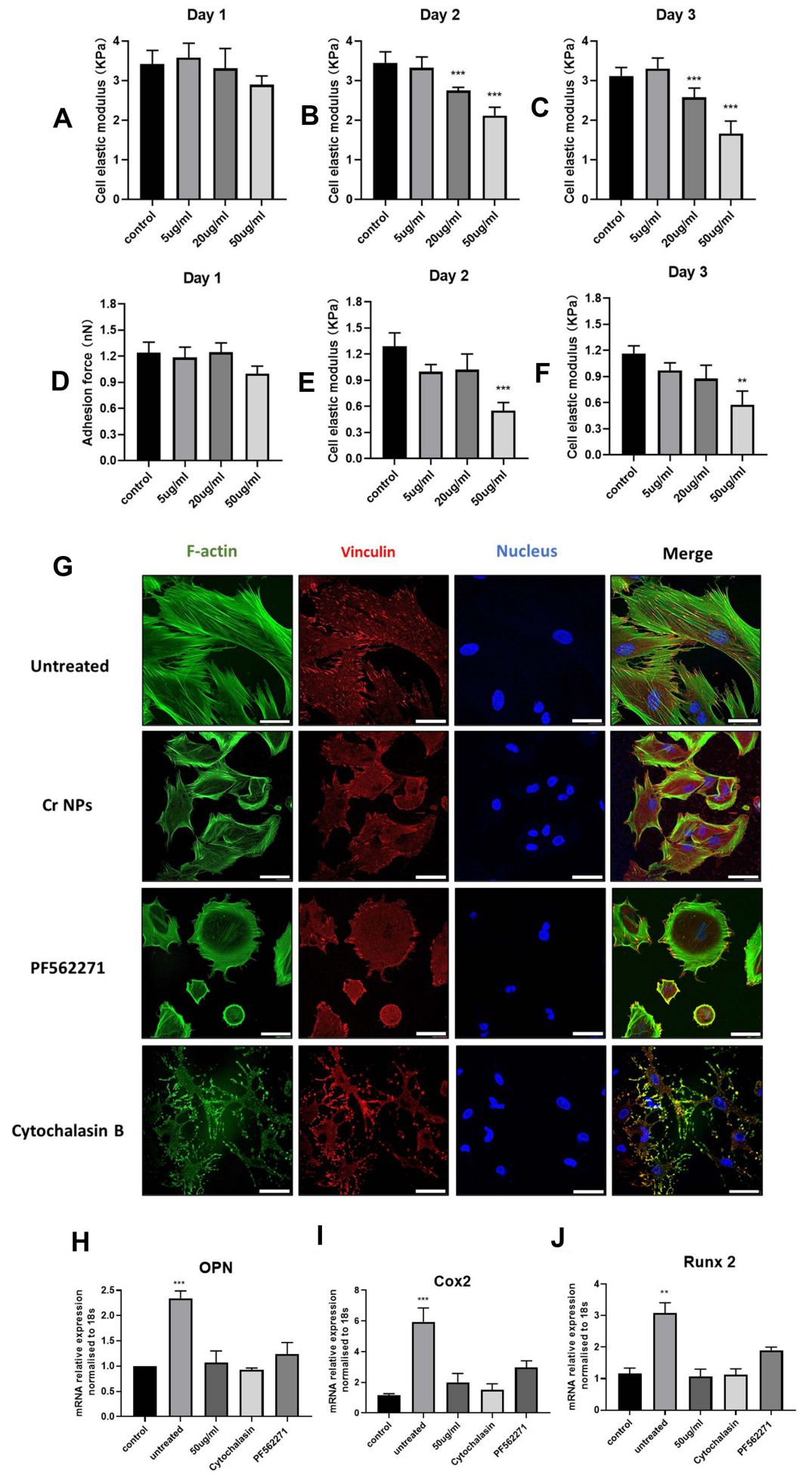

Figure 6 (A-C) Results of cell elasticity of MSCs exposed to 5-50 $\mu \mathrm{g} / \mathrm{mL}$ of CrNPs assessed by AFM. (D-F) Results of cell adhesion force of MSCs exposed to CrNPs. Data represents mean +SEM from 3 independent experiments; $n=30$, unpaired Student's $t$-test. $* *$ indicated $p<0.01$ and $* * * i n d i c a t e d ~ p<0.00 I$. (G) Immunofluorescent staining of the F-actin and vinculin from untreated MSCs and cells exposed to $50 \mu \mathrm{g} / \mathrm{mL}$ of CrNPs, Cytochalasin B $(2 \mu \mathrm{M})$ and PF56227I (5 $\mu$ M). Effect of CrNPs, Cytochalasin B and PF56227I on early mRNA expression of osteogenic genes OPN (H), Cox2 (I) and Runx2 (J) under fluid flow. Bars represent mean + SEM; Statistically significant differences are indicated relative to control (under no-flow condition) based on unpaired $t$-test, **Indicated $p<0.01$ and $* * * p<0.001$. 
loading on MSCs upregulates transcription of genes that lead to enhanced osteogenesis, which includes osteoblast differentiation and bone matrix protein production. ${ }^{33,40}$ It was showed in this study that osteogenesis of MSCs was markedly reduced due to CrNPs treatment after 2 weeks of culture under fluid flow (Figure 5D). Further, fluid flow-induced transcriptional upregulation of OPN and Cox2 that is associated with osteogenesis in MSCs was also significantly reduced by CrNPs treatment in a dose-dependent manner (Figure 5F and G).

It has been found that mechanical cues are vital for MSC differentiation and bone formation. ${ }^{41,42}$ On the other hand, the mechanical behaviour of cells is mainly controlled by their structural integrity and the organization of actin filament cytoskeleton. $^{43}$ Therefore, we applied AFM nanoindentation to investigate the mechanical responses of MSCs to CrNPs. It has been documented that the mechanical properties of the cell have a critical role in determining the fate of the cell and affect a series of cell functions. ${ }^{44,45}$ For example, MSCs could undergo neurogenic lineage differentiation on softer matrix whereas develop myogenic and osteogenic phenotypes on stiffer matrices. ${ }^{46}$ In general, MSCs displaced a reduction in cell elasticity and adhesion force as the concentration of CrNPs increased over 3 days of treatment (Figure 6). The effects were most prominent after the cells were exposed to the particles for $72 \mathrm{~h}$. Cytoskeletal remodelling or disruption has been suggested as a likely cause of the alteration in cell elastic properties and functions. This hypothesis supports our results showing that CrNPs induced structural responses via disruption of actin cytoskeleton without fatal cell damage (Figure 6G). The elastic modulus of cells is of critical implication as it has been suggested that changes to cellular stiffness are associated with many pathological alteration or disorders. ${ }^{47,48}$ Even though CrNPs may not directly influence cell proliferation and viability, they induced structural changes and affected mechanical sensing of the cells. By disrupting cellular F-actin integrity and inhibiting focal adhesion kinase (FAK) that affect the cell adhesive forces with Cytochalasin B and PF562271 (Figure 6G), which showed detrimental influence on fluid flow-induced osteogenic differentiation of MSCs (Figure 6H-J), we further revealed that the effects of CrNPs on in vivo osteogenesis and flow-induced osteogenic differentiation of MSCs could be closely associated with its interruption on cell mechanical properties.

A great number of metal joint replacement devices are implanted in patients and the demand for such procedures is fuelled by the aging population. However, the biological response to these wear debris originated from the devices is yet to be determined.

\section{Conclusion}

Our results demonstrated that CrNPs impaired cellular response to mechanical stimulus and osteogenesis in vitro and in vivo without noticeable effects on the metabolic activity of the cells. These detrimental effects could be associated with changes in the mechanical properties (cell elasticity and adhesive forces) and structural integrity of cytoskeleton. This study unravels an important effect of CrNPs released from orthopaedics implant, which adds to the current understanding of interactions of mesenchymal stem cells with metallic nanoparticles.

\section{Funding}

This work was supported in part by the National Natural Science Foundation of China (Grant Nos. 81902275 and 81802082).

\section{Disclosure}

No potential conflict of interest was reported by the authors.

\section{References}

1. Learmonth ID, Young C, Rorabeck C. The operation of the century: total hip replacement. Lancet. 2007;370(9597):1508-1519. doi:10.1016/S0140-6736(07)60457-7

2. Mahomed NN, Barrett JA, Katz JN, et al. Rates and outcomes of primary and revision total hip replacement in the United States medicare population. $J$ Bone Joint Surg Am. 2003;85(1):27-32. doi:10.2106/00004623-200301000-00005

3. Billi F, Campbell P. Nanotoxicology of metal wear particles in total joint arthroplasty: a review of current concepts. J Appl Biomater Biomech. 2010;8(1):1-6.

4. Man K, Jiang LH, Foster R, Yang XB. Immunological responses to total hip arthroplasty. J Funct Biomater. 2017;8(3):33. doi:10.3390/jfb8030033

5. Jacobs JJ, Cooper HJ, Urban RM, Wixson RL, Della Valle CJ. What do we know about taper corrosion in total hip arthroplasty? J Arthroplasty. 2014;29(4):668-669. doi:10.1016/j.arth.2014.02.014

6. Cooper HJ, Della Valle CJ, Berger RA, et al. Corrosion at the head-neck taper as a cause for adverse local tissue reactions after total hip arthroplasty. $J$ Bone Joint Surg Am. 2012;94 (18):1655-1661. doi:10.2106/JBJS.K.01352

7. Madl AK, Liong M, Kovochich M, Finley BL, Paustenbach DJ, Oberdorster G. Toxicology of wear particles of cobalt-chromium alloy metal-on-metal hip implants Part I: physicochemical properties in patient and simulator studies. Nanomedicine. 2015;11 (5):1201-1215. doi:10.1016/j.nano.2014.12.005

8. Campbell P, Ebramzadeh E, Nelson S, Takamura K, De Smet K, Amstutz HC. Histological features of pseudotumor-like tissues from metal-on-metal hips. Clin Orthop Relat Res. 2010;468(9):2321-2327. doi:10.1007/s11999-010-1372-y

9. Xia Z, Ricciardi BF, Liu Z, et al. Nano-analyses of wear particles from metal-on-metal and non-metal-on-metal dual modular neck hip arthroplasty. Nanomedicine. 2017;13(3):1205-1217. doi:10.1016/j. nano.2016.11.003 
10. Carli A, Politis A, Zukor D, Huk O, Antoniou J. Clinically significant corrosion at the head-neck taper interface in total hip arthroplasty: a systematic review and case series. Hip Int. 2015;25(1):7-14. doi:10.5301/hipint.5000180

11. Posada OM, Gilmour D, Tate RJ, Grant MH. CoCr wear particles generated from $\mathrm{CoCr}$ alloy metal-on-metal hip replacements, and cobalt ions stimulate apoptosis and expression of general toxicology-related genes in monocyte-like U937 cells. Toxicol Appl Pharmacol. 2014;281(1):125-135. doi:10.1016/j.taap.2014.09.010

12. Akbar M, Fraser AR, Graham GJ, Brewer JM, Grant MH. Acute inflammatory response to cobalt chromium orthopaedic wear debris in a rodent air-pouch model. $J \quad R$ Soc Interface. 2012;9 (74):2109-2119. doi:10.1098/rsif.2012.0006

13. Granchi D, Ciapetti G, Stea S, et al. Cytokine release in mononuclear cells of patients with Co-Cr hip prosthesis. Biomaterials. 1999;20 (12):1079-1086. doi:10.1016/S0142-9612(99)00004-6

14. Howling GI, Sakoda H, Antonarulrajah A, et al. Biological response to wear debris generated in carbon based composites as potential bearing surfaces for artificial hip joints. J Biomed Mater Res B Appl Biomater. 2003;67(2):758-764. doi:10.1002/jbm.b.10068

15. Kaufman AM, Alabre CI, Rubash HE, Shanbhag AS. Human macrophage response to UHMWPE, TiAlV, $\mathrm{CoCr}$, and alumina particles: analysis of multiple cytokines using protein arrays. $J$ Biomed Mater Res A. 2008;84(2):464-474. doi:10.1002/jbm.a.31467

16. VanOs R, Lildhar LL, Lehoux EA, Beaule PE, Catelas I. In vitro macrophage response to nanometer-size chromium oxide particles. $J$ Biomed Mater Res B Appl Biomater. 2014;102(1):149-159. doi:10.1002/jbm.b.32991

17. Pajarinen J, Lin TH, Nabeshima A, et al. Mesenchymal stem cells in the aseptic loosening of total joint replacements. J Biomed Mater Res A. 2017;105(4):1195-1207. doi:10.1002/jbm.a.35978

18. Undale AH, Westendorf JJ, Yaszemski MJ, Khosla S. Mesenchymal stem cells for bone repair and metabolic bone diseases. Mayo Clin Proc. 2009;84(10):893-902. doi:10.4065/84.10.893

19. James AW. Review of signaling pathways governing MSC osteogenic and adipogenic differentiation. Scientifica. 2013;2013:684736. doi: $10.1155 / 2013 / 684736$

20. Marie PJ, Fromigue O. Osteogenic differentiation of human marrow-derived mesenchymal stem cells. Regen Med. 2006;1 (4):539-548. doi:10.2217/17460751.1.4.539

21. Li YJ, Batra NN, You L, et al. Oscillatory fluid flow affects human marrow stromal cell proliferation and differentiation. J Orthop Res. 2004;22(6):1283-1289. doi:10.1016/j.orthres.2004.04.002

22. Xu J, Yang J, Chen J, et al. Activation of synovial fibroblasts from patients at revision of their metal-on-metal total hip arthroplasty. Part Fibre Toxicol. 2020;17(1):42. doi:10.1186/s12989-020-00374-y

23. Xu J, Nyga A, Li W, et al. Cobalt ions stimulate a fibrotic response through matrix remodelling, fibroblast contraction and release of pro-fibrotic signals from macrophages. Eur Cell Mater. 2018;36:142-155. doi:10.22203/eCM.v036a11

24. Chen S, Qin L, Wu X, et al. Moderate fluid shear stress regulates heme oxygenase-1 expression to promote autophagy and ECM homeostasis in the nucleus pulposus cells. Front Cell Dev Biol. 2020;8:127. doi:10.3389/fcell.2020.00127

25. Xu J, Yang J, Nyga A, et al. Cobalt (II) ions and nanoparticles induce macrophage retention by ROS-mediated down-regulation of RhoA expression. Acta Biomater. 2018;72:434-446.

26. Doorn PF, Campbell PA, Worrall J, Benya PD, McKellop HA, Amstutz HC. Metal wear particle characterization from metal on metal total hip replacements: transmission electron microscopy study of periprosthetic tissues and isolated particles. J Biomed Mater Res. 1998;42(1):103-111. doi:10.1002/(SICI)1097-4636(199810)42:1<103::AID-JBM13>3.0.CO;2-M
27. Catelas I, Campbell PA, Bobyn JD, Medley JB, Huk OL. Wear particles from metal-on-metal total hip replacements: effects of implant design and implantation time. Proc Inst Mech Eng $\mathrm{H}$. 2006;220(2):195-208. doi:10.1243/09544119JEIM112

28. Milosev I, Remskar M. In vivo production of nanosized metal wear debris formed by tribochemical reaction as confirmed by high-resolution TEM and XPS analyses. $J$ Biomed Mater Res A. 2009;91(4):1100-1110. doi:10.1002/jbm.a.32301

29. Catelas I, Bobyn JD, Medley JB, Krygier JJ, Zukor DJ, Huk OL. Size, shape, and composition of wear particles from metal-metal hip simulator testing: effects of alloy and number of loading cycles. $J$ Biomed Mater Res A. 2003;67(1):312-327. doi:10.1002/jbm. a. 10088

30. Madl AK, Kovochich M, Liong M, Finley BL, Paustenbach DJ, Oberdorster G. Toxicology of wear particles of cobalt-chromium alloy metal-on-metal hip implants Part II: importance of physicochemical properties and dose in animal and in vitro studies as a basis for risk assessment. Nanomedicine. 2015;11(5):1285-1298. doi:10.1016/j.nano.2015.02.006

31. Abu-Amer Y, Darwech I, Clohisy JC. Aseptic loosening of total joint replacements: mechanisms underlying osteolysis and potential therapies. Arthritis Res Ther. 2007;9(Suppl 1):S6. doi:10.1186/ar2170

32. Wang YK, Chen CS. Cell adhesion and mechanical stimulation in the regulation of mesenchymal stem cell differentiation. J Cell Mol Med. 2013;17(7):823-832. doi:10.1111/jcmm.12061

33. Chen JC, Jacobs CR. Mechanically induced osteogenic lineage commitment of stem cells. Stem Cell Res Ther. 2013;4(5):107. doi: $10.1186 /$ scrt3 18

34. Infante A, Rodriguez CI. Osteogenesis and aging: lessons from mesenchymal stem cells. Stem Cell Res Ther. 2018;9(1):244. doi:10.1186/s13287-018-0995-x

35. Posada OM, Tate RJ, Grant MH. Effects of CoCr metal wear debris generated from metal-on-metal hip implants and Co ions on human monocyte-like U937 cells. Toxicol in Vitro. 2015;29(2):271-280. doi:10.1016/j.tiv.2014.11.006

36. Klein-Nulend J, Bacabac RG, Bakker AD. Mechanical loading and how it affects bone cells: the role of the osteocyte cytoskeleton in maintaining our skeleton. Eur Cell Mater. 2012;24:278-291. doi:10.22203/eCM.v024a20

37. Cardoso L, Fritton SP, Gailani G, Benalla M, Cowin SC. Advances in assessment of bone porosity, permeability and interstitial fluid flow. J Biomech. 2013;46(2):253-265. doi:10.1016/j.jbiomech.2012.10.025

38. Hillsley MV, Frangos JA. Bone tissue engineering: the role of interstitial fluid flow. Biotechnol Bioeng. 1994;43(7):573-581. doi:10.1002/bit.260430706

39. Steward AJ, Kelly DJ. Mechanical regulation of mesenchymal stem cell differentiation. J Anat. 2015;227(6):717-731. doi:10.1111/ joa. 12243

40. Chen JC, Chua M, Bellon RB, Jacobs CR. Epigenetic changes during mechanically induced osteogenic lineage commitment. $J$ Biomech Eng. 2015;137(2):20902. doi:10.1115/1.4029551

41. Yang W, Zhong Y, He C, et al. Electrostatic self-assembly of pFe3O4 nanoparticles on graphene oxide: a co-dispersed nanosystem reinforces PLLA scaffolds. $J A d v$ Res. 2020;24:191-203. doi:10.1016/j. jare.2020.04.009

42. Burger EH, Klein-Nulend J, Veldhuijzen JP. Mechanical stress and osteogenesis in vitro. J Bone Miner Res. 1992;7(Suppl 2):S397-401. doi:10.1002/jbmr.5650071406

43. Fletcher DA, Mullins RD. Cell mechanics and the cytoskeleton. Nature. 2010;463(7280):485-492. doi:10.1038/nature08908

44. Potier E, Noailly J, Ito K. Directing bone marrow-derived stromal cell function with mechanics. J Biomech. 2010;43(5):807-817. doi:10.1016/j.jbiomech.2009.11.019 
45. Chen J, Irianto J, Inamdar S, et al. Cell mechanics, structure, and function are regulated by the stiffness of the three-dimensional microenvironment. Biophys J. 2012;103(6):1188-1197. doi:10.1016/ j.bpj.2012.07.054

46. Engler AJ, Sen S, Sweeney HL, Discher DE. Matrix elasticity directs stem cell lineage specification. Cell. 2006;126(4):677-689. doi:10.1016/j.cell.2006.06.044
47. Kawauchi K, Fujita H, Miyoshi D, Yim EK, Hirata H. Cell and molecular mechanics in health and disease. Biomed Res Int. 2017;2017:2860241. doi:10.1155/2017/2860241

48. Chen JH, Simmons CA. Cell-matrix interactions in the pathobiology of calcific aortic valve disease: critical roles for matricellular, matricrine, and matrix mechanics cues. Circ Res. 2011;108 (12):1510-1524. doi:10.1161/CIRCRESAHA.110.234237

\section{Publish your work in this journal}

The International Journal of Nanomedicine is an international, peerreviewed journal focusing on the application of nanotechnology in diagnostics, therapeutics, and drug delivery systems throughout the biomedical field. This journal is indexed on PubMed Central, MedLine, CAS, SciSearch ${ }^{\mathbb{R}}$, Current Contents ${ }^{\mathbb{R}} /$ Clinical Medicine, $^{-}$
Journal Citation Reports/Science Edition, EMBase, Scopus and the Elsevier Bibliographic databases. The manuscript management system is completely online and includes a very quick and fair peer-review system, which is all easy to use. Visit http://www.dovepress.com/ testimonials.php to read real quotes from published authors. 